\title{
Spontaneous spinal epidural haematoma: a cautionary tale
}

\author{
G. M. LORD \& N. MENDOZA \\ Charing Cross Hospital, Fulham Palace Road, London W6 8RF
}

\section{SUMMARY}

Less than two hundred cases of spontaneous spinal epidural heamatoma (SSEH) have been reported in the literature and theories as to its genesis are diverse. It is a serious condition, especially if there is a delay in diagnosis, as early treatment confers marked prognostic advantage. We present a case report of a 68 -year-old male who was diagnosed as having a spinal epidural haematoma, followed by a discussion of the possible aetiology of this condition.

\section{Clinical history and examination}

A 68-year-old man presented to an accident and emergency department (A\&E) with a 1-h history of sudden onset of neck pain radiating across both shoulders, coming on after turning the head to the left at the wheel of a motor car. There was no history of trauma or previous spinal pathology.

On examination, there was marked reduction in active cervical spine movement in all directions due to pain.

There was some paraspinal tenderness on the left at the level of the fifth cervical vertebra, and in the region of his left supraspinatus muscle. The pain in his neck region had decreased significantly. He was known to be hypertensive, for which he was on treatment with captopril. His blood pressure was 210/110. Neurological examination was normal.

A provisional diagnosis of wry neck was made, and he was to be discharged home with a soft cervical collar, anti-inflammatory medication and an appointment for physiotherapy.

Prior to discharge, $1 \mathrm{~h}$ after entering the department, he was reviewed after he complained of altered sensation in his left arm and weakness in the right arm and right leg.

Upon re-examination, there was evidence of a progressive tetraparesis. He had 
absent biceps and supinator reflexes in the right arm, and increased knee and ankle jerks with an upgoing plantar response in the left leg. Power in the upper limb on the right was $2 / 5$ in all groups and power in the ipsilateral lower limb was $1 / 5$ in all groups. Sensation on the right was preserved. On the left, a dermatomal sensory loss could not be demonstrated, although a C4/T2 sensory level was found. Over the next $15 \mathrm{~min}$ his neurological deficit progressed in a stepwise fashion to a position of almost total tetraplegia, with some sparing of power in the left arm.

In view of the above findings a diagnosis of cervical cord compression or infarction was made. Dexamethasone (16 mg i.v.) was administered, and a neurosurgical opinion requested.

\section{Investigation and treatment}

Spinal radiographs demonstrated changes consistent with cervical spondylosis but were otherwise normal, with no evidence of instability. Cervical CT myelography was performed, demonstrating the presence of a SSEH extending from C4 to C5 (Fig. 1). A decompressive cervical laminectomy from C3-C6 and evacuation of the haematoma was performed.

Haematological investigations indicated that there was no evidence of coagulopathy and no thrombocytopaenia.

Post-operatively, the patient made a complete recovery and was discharged 10 days following admission.

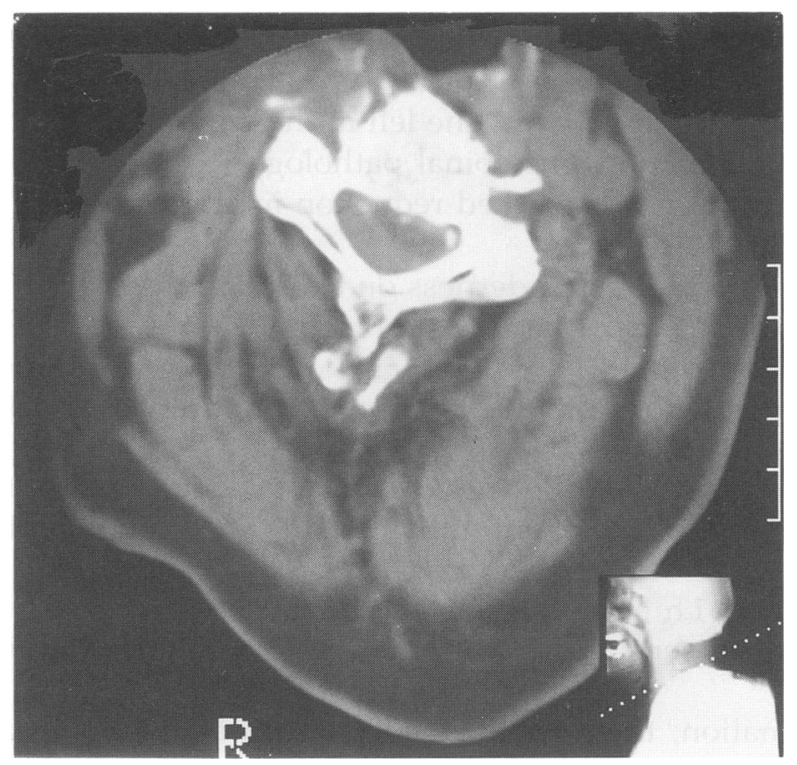

Fig. 1. CT Myelogram at the level of the fifth cervical vertebra, demonstrating an extradural haematoma compressing the spinal cord. 


\section{DISCUSSION}

SSEH is a rare condition, there being less than two hundred cases reported in the literature over the past 50 years.

Initially it was described towards the end of the last century, and many theories as to the aetiology of the condition have been postulated.

It is well understood and accepted that major trauma (for example, vertebral fracture), iatrogenic or pathological bleeding diathesis, and vertebral body haemangioma can all be causative factors (Cube, 1962). However the condition of SSEH, by definition, excludes these aetiological categories.

SSEH has been variously associated with minor trauma (Cooper, 1967), hypertension (Ainslie, 1958), coughing, sneezing, voiding and spontaneous bleeding from a vascular anomaly (Foo \& Rossier, 1981).

In a recent review (Groen \& Ponssen, 1990), however, it has been suggested that the factors above play little part in the genesis of SSEH. They point to the fact that hypertension is no more prevalent in patients with SSEH than in age matched controls. It was also noted that the incidence of a vascular anomaly in patients with SSEH may be overestimated, as it can be confused with the cluster-type dural artery, and that the evidence seems to point to bleeding from the posterior internal vertebral venous plexus, presumably because of either intrinsic or acquired structural defects.

The most important factor in determining outcome is the time elapsed between onset of symptoms and treatment (McQuarrie, 1978), which is decompressive laminectomy and evacuation of the haematoma.

The major obstacle lies in suspecting the diagnosis, especially in cases where there is a temporal disparity between the onset of symptoms (i.e. pain) and the appearance of hard neurological signs, which has been well described (Liebeskind et al., 1975). The diagnosis must be suspected in any patient presenting with acute onset severe neck or back pain, with a history of minimal or no trauma, when associated with radicular pain or neurological signs consistent with cord compression

The differential diagnosis lies between an acute prolapsed inter-vertebral disc, a vascular occlusion and a transverse myelopathy. The investigation of choice is CT-myelography, which may be normal in the latter two cases and diagnostic in the case of a prolapsed intervertebral disc and SSEH.

\section{CONCLUSION}

In view of the fact that symptoms and signs may be separated from each other by several hours, it is important to maintain a high index of suspicion of SSEH, as early intervention usually results in total recovery of cord function and the medicolegal consequences of a missed diagnosis of SSEH could be potentially disastrous. 


\section{ACKNOWLEDGEMENTS}

We would like to thank $\mathrm{Mr} \mathrm{H}$. T. Millington for allowing us to report his patient.

\section{REFERENCES}

Ainslie J. P. (1958) Paraplegia due to spontaneous extradural or subdural haemorrhage. British Journal of Surgery 45, 565-567.

Cooper D. W. (1967) Spontaneous spinal epidural haematoma. Journal of Neurosurgery 26, $343-345$.

Cube H. M. (1962) Spinal extradural haemorrhage. Journal of Neurosurgery 19, 171-172.

Foo D. \& Rossier A. B. (1981) Preoperative neurological status in predicting surgical outcome of spinal epidural haematomas. Surgical Neurology 15, 398-401.

Groen R. J. M. \& Ponssen H. (1990) The spontaneous spinal epidural haematoma: a study of etiology. Journal of Neurological Science's 98, 121-138.

Liebeskind A. L., Schwartz K. S., Coffey E. L. \& Beresford H. R. (1975) Spinal epidural haematoma with delayed appearance of neurological signs. Neuroradiology 8, 191-193.

McQuarrie I. G. (1978) Recovery from paraplegia caused by spontaneous spinal spidural haematoma. Neurology 28, 224-228. 